\title{
Editorial \\ PARP inhibitors and the treatment of breast cancer: beyond BRCA1/2?
}

\author{
Kristine M Frizzell ${ }^{1,2}$ and W Lee Kraus ${ }^{1-3}$
}

1Department of Molecular Biology and Genetics, Cornell University, 465 Biotechnology Building, Ithaca, NY 14853, USA
2Graduate Field of Biochemistry, Molecular and Cell Biology, Cornell University, 465 Biotechnology Building, Ithaca, NY 14853, USA

${ }^{3}$ Department of Pharmacology, Weill Medical College of Cornell University, 1300 York Avenue, New York, NY 10021, USA

Corresponding author:W Lee Kraus,wlk5@cornell.edu

Published: 26 November 2009

Breast Cancer Research 2009, 11:111 (doi:10.1186/bcr2451)

This article is online at http://breast-cancer-research.com/content/11/6/111

(c) 2009 BioMed Central Ltd

See related research by Inbar-Rozensal et al., http://breast-cancer-research.com/content/11/6/R78

\begin{abstract}
Poly(ADP-ribose) polymerase (PARP) inhibitors have been explored as therapeutic agents for the treatment of hereditary breast and ovarian cancers harboring mutations in BRCA1 or BRCA2. In a new study, Inbar-Rozensal and colleagues show that phenanthridine-derived PARP inhibitors promote cell cycle arrest and cell death in breast cancer cells lacking BRCA1 and BRCA2 mutations and prevent the growth of tumors from xenografts of these cells in immunocompromised mice. These results suggest a potential broader utility of PARP-1 inhibitors in the treatment of breast cancer, although further mechanistic studies are needed.
\end{abstract}

Recent studies have suggested that chemical inhibitors of poly(ADP-ribose) polymerases (PARPs) might be effective as therapeutic agents for the treatment of hereditary breast and ovarian cancers harboring mutations in $B R C A 1$ or BRCA2. In a recent article from the Cohen-Armon group published in Breast Cancer Research, Inbar-Rozensal and colleagues [1] provide evidence that certain PARP inhibitors might also inhibit the growth and promote the death of non-hereditary breast cancer cells lacking mutations in BRCA1 or BRCA2. These intriguing results might lead the way to new approaches for treating a broad spectrum of breast cancer subtypes.

PARPs comprise a family of enzymes that catalyze the polymerization of poly(ADP-ribose) chains on target proteins, thereby modifying the activity of those proteins [2]. Nuclear PARPs, such as PARP-1 and PARP-2, play key roles in genome maintenance, cell death, inflammatory responses, and the control of gene expression programs [2,3]. PARP enzymatic activity increases in response to various cellular stresses [2,3]. Given the central role of PARPs in vital cellular processes as well as disease states, chemical inhibitors of PARP have been explored as therapeutic agents for a wide variety of diseases, including cancer [4,5].
Increasing evidence has linked PARP-1 to breast cancer. For example, PARP-1-deficient mice exhibit increased spontaneous mammary carcinoma formation, the latency of which is increased by mutations in $p 53$ [6]. In addition, PARP activity in human peripheral blood lymphocytes has been linked with breast cancer [7] and low levels of PARP-1 gene expression are associated with increased genetic instability in breast cancer [8]. Furthermore, certain polymorphisms in PARP-1 may contribute to the development of breast cancer and influence the effectiveness of hormone therapies [9]. Interestingly, PARP inhibition (a) sensitizes p53-deficient breast cancer cells to doxorubicin-induced apoptosis [10] and (b) selectively kills breast cancer cells with hereditary inactivating mutations in BRCA1 and BRCA2, which encode proteins critical for DNA repair by homologous recombination [5]. Finally, the PARP inhibitor, olaparib, has anti-tumor activity in breast and ovarian cancers containing BRCA1 and BRCA2 mutations at safely administrable doses with minimal side effects [11].

Building upon these exciting studies, Inbar-Rozensal and colleagues [1] show that phenanthridine-derived PARP inhibitors (for example, PJ-34) promote cell cycle arrest at $\mathrm{G}_{2} / \mathrm{M}$ and cell death in breast cancer cell lines lacking BRCA1 and BRCA2 mutations (that is, MCF-7 and MDA-MB-231). These effects were evident even after a short (6-hour) treatment, and no recovery was observed after drug removal. In contrast, although a transient cell cycle arrest was also observed in normal breast epithelial cells (that is, MCF-10A) and mouse embryo fibroblasts, recovery was apparent within hours, even with continued drug exposure. Furthermore, in immunocompromised nude mice, PJ-34 prevented the growth of tumors from subcutaneous xenografts of MCF-7 or MDA-MB-231 cells. From these studies, the authors 
conclude that phenanthridine-derived PARP inhibitors cause cell cycle arrest and subsequent cell death in non-hereditary breast cancer cells. Additional studies are needed, however, to fully explore the activity of other structural classes of PARP inhibitors. If these results translate into similar effects in clinical studies, this would truly be a remarkable finding with great therapeutic potential.

As with any promising initial study, many questions remain, especially regarding the mechanism of action. Based on the previous results with the BRCA1/2-deficent cells, the assumption is that the ultimate target of the PARP inhibition is a DNA repair pathway. Although MCF-7 and MDA-MB-231 cells are apparently wild-type for BRCA1 and BRCA2, they may harbor mutations in genes encoding other DNA repair and checkpoint proteins (for example, Rad51 and Chk1/2) that could render them sensitive to PARP inhibitors. Importantly, nuclear PARPs, such as PARP-1 and PARP-2, also play key roles in gene regulation [2,3], so transcriptional effects cannot be ruled out as the cause of PARP inhibitor sensitivity. Experiments with depletion of specific PARPs will help to determine the relevant targets.

Inbar-Rozensal and colleagues suggest a possible mechanism underlying $\mathrm{G}_{2} / \mathrm{M}$ arrest by the PARP inhibitors through signal transduction pathways involving cell cycle proteins (for example, p21, cyclins, and cdc2) and extracellular signalregulated kinase (ERK)-dependent kinase cascades. This is a reasonable hypothesis given the involvement of these pathways in cell cycle progression and proliferation in a variety of cancers, but additional experimental support is needed. The Cohen-Armon group [12] previously demonstrated that a signaling pathway involving ERK2 can support sustained DNA-independent activation of PARP-1, which is tied to signal-dependent chromatin modulation and gene regulation.

Interestingly, PARP-1 expression levels are increased in triple-negative breast cancer (that is, those that do not express estrogen receptor [ER], progesterone receptor [PR], and the human epidermal growth factor receptor HER2 [13]). Although MCF-7 cells and MDA-MB-231 cells differ in their expression of ER and PR (that is, MCF-7 cells express both ER and PR whereas MDA-MB-231 cells do not), neither cell line expresses HER2 [14]. HER2 is a cell membrane receptor tyrosine kinase involved in signaling pathways leading to cell proliferation, including those mediated by ERKs [15]. Functional connections between HER2, ERKs, and PARP-1 might play a role in determining the sensitivity of breast cancer subtypes to PARP inhibitors. Comparing the effects of phenanthridine-derived PARP inhibitors in MCF-7 and MDAMB-231 cells with forced expression of HER2 would be informative in this regard.

In summary, the intriguing results of Inbar-Rozensal and colleagues point to a broader utility of PARP inhibitors in the treatment of breast cancer beyond hereditary BRCA1and BRCA2-deficient types. Further mechanistic studies are needed, however, to understand the full spectrum of breast cancer types for which chemical inhibition of PARPs might be therapeutically beneficial. In addition, clinical studies will be required to assess the efficacy in situ. Nonetheless, this study deserves attention and should stimulate much-needed further research in this area.

\section{Competing interests}

The authors declare that they have no competing interests.

\section{References}

1. Inbar-Rozensal D, Castiel A, Visochek L, Castel D, Dantzer F, Izraeli S, Cohen-Armon M: A selective eradication of human nonhereditary breast cancer cells by phenanthridine-derived polyADP-ribose polymerase inhibitors. Breast Cancer Res 2009, 11:R78.

2. Kim MY, Zhang T, Kraus WL: Poly(ADP-ribosyl)ation by PARP-1: 'PAR-laying' NAD' into a nuclear signal. Genes Dev 2005, 19: 1951-1967.

3. Kraus WL: Transcriptional control by PARP-1: chromatin modulation, enhancer-binding, coregulation, and insulation. Curr Opin Cell Biol 2008, 20:294-302.

4. Kling J: PARP inhibitors blaze a trail in difficult-to-treat cancers. Nat Biotechnol 2009, 27:784-786.

5. Drew $\mathrm{Y}, \mathrm{Calvert} \mathrm{H}$ : The potential of PARP inhibitors in genetic breast and ovarian cancers. Ann N Y Acad Sci 2008, 1138: 136-145.

6. Tong WM, Yang YG, Cao WH, Galendo D, Frappart L, Shen Y, Wang ZQ: Poly(ADP-ribose) polymerase-1 plays a role in suppressing mammary tumourigenesis in mice. Oncogene 2007, 26:3857-3867.

7. Pero RW, Roush GC, Markowitz MM, Miller DG: Oxidative stress, DNA repair, and cancer susceptibility. Cancer Detect Prev 1990, 14:555-561.

8. Bieche I, de Murcia G, Lidereau R: Poly(ADP-ribose) polymerase gene expression status and genomic instability in human breast cancer. Clin Cancer Res 1996, 2:1163-1167.

9. Cao WH, Wang X, Frappart L, Rigal D, Wang ZQ, Shen Y, Tong WM: Analysis of genetic variants of the poly(ADP-ribose) polymerase-1 gene in breast cancer in French patients. Mutat Res 2007, 632:20-28.

10. Muñoz-Gámez JA, Martín-Oliva D, Aguilar-Quesada R, Cañuelo A Nuñez MI, Valenzuela MT, Ruiz de Almodóvar JM, De Murcia G, Oliver FJ: PARP inhibition sensitizes p53-deficient breast cancer cells to doxorubicin-induced apoptosis. Biochem J 2005, 386:119-125.

11. Fong PC, Boss DS, Yap TA, Tutt $A$, Wu $P$, Mergui-Roelvink $M$, Mortimer P, Swaisland H, Lau A, O'Connor MJ, Ashworth A, Carmichael J, Kaye SB, Schellens JH, de Bono JS: Inhibition of poly(ADP-ribose) polymerase in tumors from BRCA mutation carriers. N Engl J Med 2009, 361:123-134.

12. Cohen-Armon M, Visochek L, Rozensal D, Kalal A, Geistrikh I, Klein R, Bendetz-Nezer S, Yao Z, Seger R: DNA-independent PARP-1 activation by phosphorylated ERK2 increases Elk1 activity: a link to histone acetylation. Mol Cell 2007, 25:297308.

13. Tuma RS: PARP inhibitors: will the new class of drugs match the hype? J Nat/ Cancer Inst 2009, 101:1230-1232.

14. Neve RM, Chin K, Fridlyand J, Yeh J, Baehner FL, Fevr T, Clark L, Bayani N, Coppe JP, Tong F, Speed T, Spellman PT, DeVries S, Lapuk A, Wang NJ, Kuo WL, Stilwell JL, Pinkel D, Albertson DG, Waldman FM, McCormick F, Dickson RB, Johnson MD, Lippman M, Ethier S, Gazdar A, Gray JW: A collection of breast cancer cell lines for the study of functionally distinct cancer subtypes. Cancer Cell 2006, 10:515-527.

15. Lohrisch C, Piccart M: HER2/neu as a predictive factor in breast cancer. Clin Breast Cancer 2001, 2:129-135. 